\title{
Epidemiology of Gall Bladder Carcinoma As An Unexpected His- tologic Diagnosis in Patients Undergoing Cholecystectomy for Cholelithiasis. -A Prospective Study of 300 Cases.
}

\author{
Ahi Kuldip Singh ${ }^{1}$,Sampley Sunil ${ }^{1}$,Jain Aditya ${ }^{2}$, Kumar Avnish $^{2}$ \\ ${ }^{I}$ Department Of Surgery, Government Medical College, Patiala, India. \\ ${ }^{2}$ Department Of Physiology, Government Medical College, Patiala, India
}

\begin{abstract}
Biliary tract carcinomas which include cancer of the gall bladder and intrahepatic and extrahepatic biliary tree are relatively infrequent but highly lethal diseases that are notoriously difficult to diagnose and treat.

Objective: To study the relation of gallstones and malignant histopathological changes in gallbladder with correlation of size and no. of gallstones in causation or predisposition to gallbladder carcinoma.

Methods: A prospective study was conducted at the Govt. Medical College, Patiala, on 300 patients with cholecystectomy for cholelithiasis and extensive review was done with special reference to the histopathological findings and correlation of size and no. of gallstones in causation or predisposition to gallbladder carcinoma Results: Out of 300 cholecystectomies performed during the period of study, most of the patients belonged to the age group of 31-50yrs with M: F ratio of 1:5 and the size of the largest stone measured was $<3 \mathrm{~cm}$ in $88 \%$ and $>3 \mathrm{~cm}$ in $22 \%$. Patients diagnosed to have gallbladder carcinoma in our series had size between $2-2.9 \mathrm{~cm}$ in diameter. 4 patients (1.15\%) were diagnosed to have gall bladder carcinoma on histopathology.

Conclusion: Cholelithiasis is a disease more prevalent in females in age group of 31-50years with multiple gallstones. Early onset of gallstone disease, especially in women, and late presentation of patients for surgical treatment result in prolonged exposure of the gallbladder to gallstones, increasing size of gallstone with increasing age and various complications of long standing cholelithiasis, all are risk factors for gallbladder carcinoma.
\end{abstract}

Keywords: gall bladder, carcinoma, cholecystectomy, cholelithiasis, histopathological

\section{Introduction}

Gall bladder cancer was first described in 1777 (Nevin et al, 1976). More than 200 years later, late diagnosis and absence of effective treatment for many patients remain typical feature of this disease. Epidemiologic knowledge regarding GB carcinoma is scarce for several reasons:

a) The pre-operative diagnosis is still very inaccurate, with many false positives and false negatives; therefore, information based on mortality data is unreliable.

b) An operation does not always provide the correct diagnosis, since the surgeon may be reluctant to biopsy the tumor if it is inoperable.

c) Even at autopsy it may be difficult to determine the primary location of the tumor. (Hart et al, 1971)

The prevalence of carcinoma in the gallbladder ( $\mathrm{Ca} \mathrm{GB}$ ) is greatly variable in different parts of the world. In the United States Of America, Singapore and Nigeria; it is an uncommon cancer with an incidence of 2.5 per 100000 population. In contrast, Chile has a much higher incidence of 7.5 per 100000 population, especially in American Indian females. The Indian Council of Medical Research Cancer Registry has recorded an incidence of 4.5 per 100000 population in males and 10.1 per 100000 population in females of northern parts of India, and 1.2 per 100000 population in females of the southern parts of India. The highest incidence of $\mathrm{CaGB}$ in India has been seen along the Ganges delta.

\section{Material And Methods}

This study was conducted prospectively in the Department of General Surgery and department of Pathology, Govt. Medical College, Patiala. Three hundred patients with gallstones satisfying the selection and exclusion criteria, undergoing cholecystectomy were included in this study.

Inclusion criteria

1. Age between 16 years- 70 years.

2. Symptoms consistent with biliary colic

3. Fit for general anaesthesia

4. No clinical biochemical or ultrasonographic evidence of CBD stones 
5. Informed consent

\section{Exclusion criteria}

1. Age below 16 years.

2. Acute Cholecystitis

3. Acute Pancreatitis

4. Previous abdominal surgery

5. Pregnancy

6. Prolonged administration of NSAIDS or other analgesics

7. Cirrhosis

8. Bleeding disorders

9. Choledochal cyst

10. Porcelain gall bladder

11. Gall bladder polyp

12. Anomalous pancreaticobiliary duct junction

13. Carcinogens e.g. nitrosamines

The removed gallbladder was inspected for the outer surface and then incised to remove all the gallstones and examine the mucosa. The number and size of the gallstones were recorded. The resected specimens of gallbladder with calculi of 300 patients were submitted to Department Of Pathology and subjected to histopathological examination.

\section{Observation and Results}

This prospective study was conducted in Rajindra Hospital, Patiala on 300 patients diagnosed to have cholelithiasis and had undergone cholecystectomy. Out of the 300 patients with cholelithiasis, 250 were females and 50 were males (Male: Female ratio 1:5). The mean age of patients was 43.9 years.

Table -I: Sex Distribution Of Cholelithiasis

\begin{tabular}{|l|c|c|}
\hline Sex & No. of patients & $\%$ \\
\hline Male & 50 & 16.66 \\
\hline Female & 250 & 83.33 \\
\hline
\end{tabular}

Table- 2: Age And Sex Distribution In The Patients With Gallstone Disease

\begin{tabular}{|c|c|c|c|l|}
\hline Age Group & No. of patients & Male & Female & $\%$ \\
\hline $21-30$ & 57 & 6 & 51 & 19 \\
\hline $31-40$ & 76 & 9 & 67 & 25.33 \\
\hline $41-50$ & 91 & 16 & 75 & 30.33 \\
\hline $51-60$ & 48 & 8 & 40 & 16 \\
\hline $61-70$ & 26 & 10 & 16 & 8.66 \\
\hline $71-80$ & 1 & 1 & - & 0.33 \\
\hline $81-90$ & 1 & - & 1 & 0.33 \\
\hline Total & 300 & 50 & 250 & \\
\hline
\end{tabular}

Table-2 gives an account of age and sex distribution which reveals that cholelithiasis is a disease of age group of 31-50 year with more than $50 \%$ of all patients lying within this age group and majority of the female patients belonged to this age group. The oldest patient in the study was an $85 \mathrm{yr}$ old female.

Table- 3: Frequency Of Multiple And Single Gall Stones

\begin{tabular}{|l|l|l|}
\hline No. of gallstones & No. of patients & $\%$ \\
\hline Multiple & 238 & 79.33 \\
\hline Single & 62 & 20.66 \\
\hline
\end{tabular}

Multiple stones were found in 238 patients and single stone in 62 patients. Majority of the specimens having multiple stones were from patients of higher age group.

Table- 4: Size Of The Largest Stone

\begin{tabular}{|l|l|l|}
\hline Size of stone & No. of Specimens & $\%$ \\
\hline$<3 \mathrm{~cm}$ & 264 & 88 \\
\hline$>3 \mathrm{~cm}$ & 36 & 22 \\
\hline
\end{tabular}


The size of the largest gallstone was measured with vernier callipers and 264 specimens had stone size of less than $3 \mathrm{~cm}$. Remaining 36 gallstones had size of largest stone of more than $3 \mathrm{~cm}$. Most of the size of the stone in our specimens was between $1-2 \mathrm{~cm}$.

Table-5: Lesions Observed On Microscopic Examination In Resected Gallbladders

\begin{tabular}{|l|l|l|}
\hline Histopathological findings & No. of specimens & $\%$ \\
\hline Acute cholecystitis & 32 & 10.66 \\
\hline Chronic cholecystits & 247 & 82.33 \\
\hline Hyperplasia & 17 & 5.66 \\
\hline Metaplasia & - & - \\
\hline Dysplasia & - & - \\
\hline Carcinoma in situ & - & - \\
\hline Carcinoma & 4 & 1.33 \\
\hline
\end{tabular}

The histopathological results of 300 specimens showed acute cholecystitis in 32(10.66\%), chronic cholecystitis in 247(82.33\%), hyperplasia in 17(5.66\%) and carcinoma in 4(1.33\%). Patients whose gallbladder on histopathology showed acute inflammatory process did not have any of the acute symptoms at the time of presentation. No case of metaplasia, dysplasia or carcinoma in situ was detected amongst the 300 patients operated for cholelithiasis.

\section{Discussion}

Carcinoma of the gallbladder is the most common malignancy of the biliary tract. When compared with the worldwide incidence of hepatocellular carcinoma (HCC), gallbladder carcinoma accounts for less than $10 \%$ of the annual cases of primary hepatobiliary cancer. Over the past three decades, there appears to be a slight increase in the incidence of gallbladder carcinoma in, but this may be ascribed to more thorough reporting mechanisms rather than to a true increase in incidence. Although the youngest reported case of gall bladder carcinoma occurred in an 11years old Navajo girl4,

the peak incidence of gall bladder carcinoma is observed in the 6th and 7th decades of life. The incidence of carcinoma gall bladder in India ranges from 1.01 per 100000 for males to 10.1 per 100000 for females (ICMR 1996) but the actual number may be much more in the endemic zones of Western Bihar and Eastern Uttar Pradesh where it is the third commonest malignancy of the alimentary tract (Shukla et al 1985). In a study, it was observed that the female population had 2.7 times more cholelithiasis, and 7.9 times more cancer than the male population, and the cancer incidence increases progressively with age, especially after the 5 th decade (Csendes et al, 1991).Khanna et al in 2003 reported incidence of gallbladder carcinoma as 2.3 and 1.01 per 100,000 in female and male population respectively. It is 10 times more frequent in North compared to South India. Population-based data indicate that the incidence of gallbladder cancer is relatively high in northern Indian cities14 (Uttar, Pradesh, Bihar, Orissa,

West Bengal, and Assam). The incidence of carcinoma gallbladder varies widely in different studies. Strauch (1960), Bossart and others (1962) reported incidence of carcinoma gallbladder as $1.4 \%$ and $0.82 \%$ respectively. The incidence in our study, $1.33 \%$ thus comparable to the other studies. How ever the behaviour of this malignancy in our population as reported is higher than the western reported studies but mimics with local reported studies.Xu and Zou (2007) studied different clinicopathological characteristics between unsuspected gallbladder carcinoma and preoperatively diagnosed gallbladder carcinoma, such as in primary location, malignant degree and incidence of coexistence with cholecystolithiasis. The risk of carcinoma gall bladder in patients with gall stones may be increased 4 to 7 times (Nervi et al, 1988) and patients with gallstones $>3 \mathrm{~cm}$ in diameter have a much higher risk (Diehl 1983).

Khoo J.J. et al (2008) conducted an audit of 112 cholecystectomies to review cases of primary carcinoma of gallbladder. There were nine cases of primary carcinoma of gallbladder. Six were females and 3 males. Their ages ranged from 27 to 81 years.Experimental studies have demonstrated chronic irritation of gallbladder mucosa by using different materials such as gallstones, pebbles, pitch, linonin, glass beads etc. (Piehler et al, 1978). It has been shown that chronic trauma and inflammation can induce epithelial dysplasia, carcinoma in situ and invasive cancer (Kijima et al, 1989, Dowling \& Kelly, 1986) but a cause and effect relation has not been unequivocally proved. Furthermore, one would expect squamous carcinoma to develop as a result of chronic irritation whereas it is adenocarcinoma which is the commonest histological type of carcinoma gallbladder seen in over $90 \%$ of cases. Thus, clinical data does not establish a definitive causative role for gallstones in the development of carcinoma although the association is sufficiently frequent to suggest common antecedents or at least a facilitative role 


\section{Summary And Conclusion}

Analysis of 300 patients admitted in surgical ward, Rajindra Hospital diagnosed to have cholelithiasis was done:

- Majority of the patients of gallstone disease were females (83.33\%) with M: F ratio of 1:5 with mean age of $43.9 \mathrm{yrs}$.

- The commonest symptom of presentation was right hypochondrium pain (90\%) and dyspepsia. There was no patient presenting with jaundice.

- Multiple gallstones were found in majority of cases (79.33\%) and single stone accounting for $22.66 \%$ showing more prevalence of multiple gallstones.

- The size of the largest stone measured was $<3 \mathrm{~cm}$ in $88 \%$ and $>3 \mathrm{~cm}$ in $22 \%$. Patients diagnosed to have gallbladder carcinoma in our series had size between $2-2.9 \mathrm{~cm}$ in diameter.

- On gross examination most of the specimens showed normal serosal surface with congestion of the mucosa. The gallbladder wall was thickened in $61 \%$ of specimens showing the effect on chronic inflammation.(Figure -1,2)

- The histopathological findings revealed that $82.33 \%$ of specimens showed chronic inflammation and 10.66 $\%$ had acute inflammatory reaction. There was evidence of frank carcinoma in 4(1.33\%) specimens.(Figure $-3,4)$

- The age of the patients diagnosed as having carcinoma gallbladder was more than 50yrs of age in 3 patients with oldest patient being $85 \mathrm{yr}$ old and youngest patient $38 \mathrm{yr}$ old.

Present study highlights the importance of careful gross and histopathologic evaluation of gallbladders removed with history of chronic and acute cholecystitis. To conclude cholelithiasis is a disease more prevalent in females in age group of 31-50years with multiple gallstones. Early onset of gallstone disease, especially in women, and late presentation of patients for surgical treatment result in prolonged exposure of the gallbladder to gallstones, increasing size of gallstone with increasing age and various complications of long standing cholelithiasis, all are risk factors for gallbladder carcinoma.

\section{References}

[1]. Csendes A, Becerra M, Smok G, Medina E, Maluenda F, Morales E. Prevalence of gallbladder neoplasms in cholecystectomies. Rev Med Chil. 1991 Aug;119(8):887-90.

[2]. Diehl AK. Gallstone size and the risk of gallbladder cancer. JAMA, 1983; 250: 2323-2326.

[3]. Dowling GP, Kelly JK. The histogenesis of adenocarcinoma of the gallbladder. Cancer 1986, 58: 1702-1708.

[4]. Hart K, Modan B, Shani M. Cholelithiasis in etiology of gallbladder neoplasms. Lancet 1971, 1: 1151-1153.

[5]. Khanna R, Chansuria R, Kumar M, Shukla HS. Histological changes in gallbladder due to stone disease. Indian J Surg 2006;68:201-4.

[6]. Khoo, Joon Joon, and Nurul Akmar Misron. A clinicopathological study of nine cases of gallbladder carcinoma in 1122 cholecystectomies in Johor, Malaysia. Malaysian Journal of Pathology, 30 (1). pp. 21-26.

[7]. Kijima H, Watanabe H, Iwafuchi M, Ishihara N. Histogenesis of gallbladder carcinoma and microcarcinoma. Acta Path Jpn 1989, 39: 235 .

[8]. Nervi F, Duarte I, Gomez G et al. Frequency of gallbladder cancer in Chile. Int J Cancer 1988, 41: 657-660

[9]. Nevin JE, Moran TJ, Kay S, Ring R. Carcinoma of the gallbladder. Staging, treatment and prognosis. Cancer 1976, 37 : 141-148.

[10]. Shukla VK, Khandelwal C, Roy SK, Vaidya MP. Primary carcinoma of the gallbladder: A review of a 16 year period at the University Hospital. J. Surg Oncol 1985, 28: 32-35.

[11]. Strauch G O. primary carcinoma of gallbladder. 10 years cumulative review of American Literature Surgery 1960;47:368.

[12]. Xu LN, Zou SQ, A clinicopathological analysis in unsuspected gallbladder carcinoma: a report of 23 cases. World J Gastroenterol, 2007 Mar 28; 13(12):1857-60; discussion 1860-1.

Figure 1:

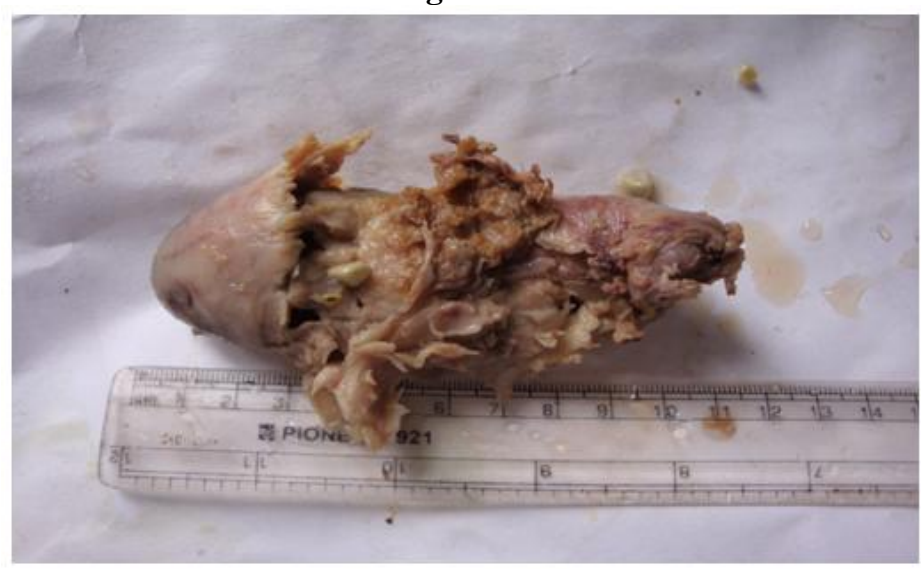


Figure 2:

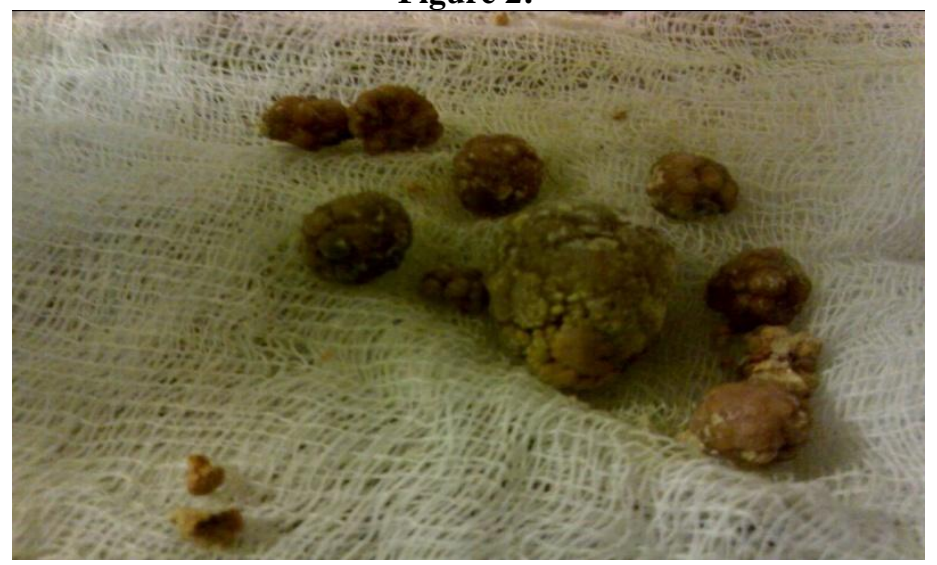

Figure 3:

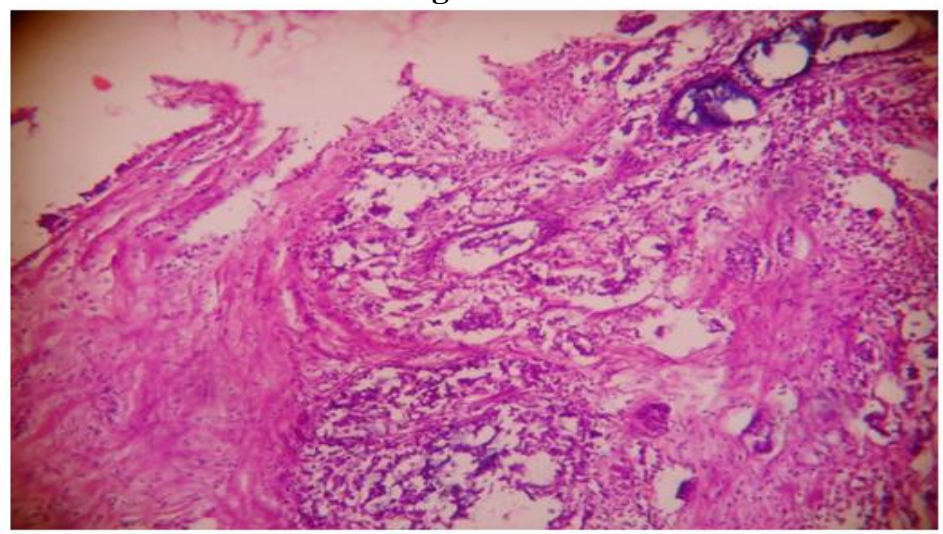

Figure 4

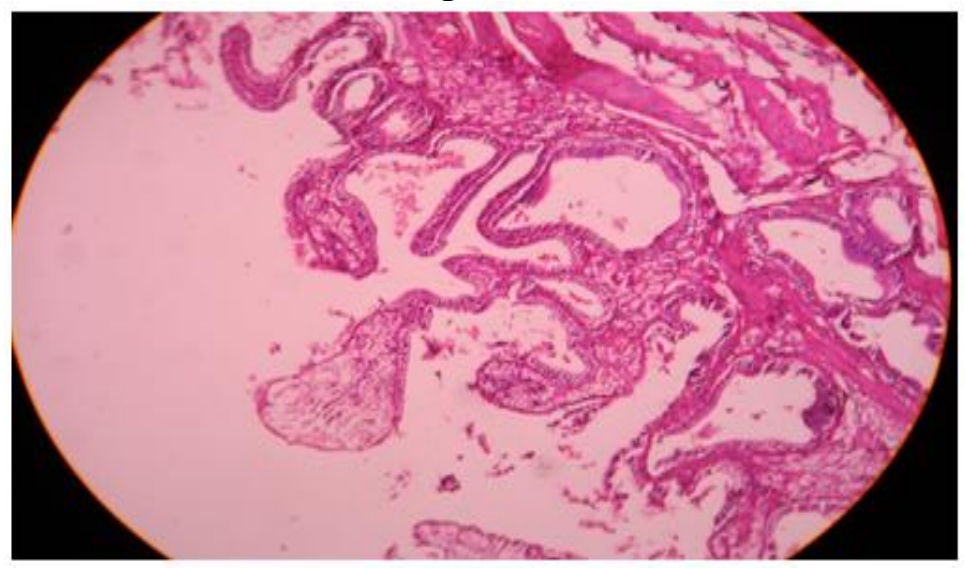

